\title{
Super-hydrophobic and super-wetting surfaces: Analytical potential?
}

\author{
G. McHale, N. J. Shirtcliffe and M. I. Newton
}

School of Science, The Nottingham Trent University, Clifton Lane, Nottingham NG11 8NS, UK; Tel: 44115
8483383; E-mail: glen.mchale@intu.ac.uk

This article appeared in the Analyst (http://www.rsc.org/Publishing/Journals/AN/article.asp?doi=b400567h).

Copyright $\mathbb{C} 2004$ Royal Society of Chemistry

Roughening or texturing surfaces provides super-liquid repellent or film forming properties without alteration of the surface chemistry. These surfaces are easy to produce, can amplify wetting properties and can be either "sticky" or "slippy" to liquids. Their use as waterrepellent coatings is established, but their potential for use in microfluidics and sensor applications remains largely unfulfilled. This article explains several key ideas and suggests why there may be potential for analytical applications.

\section{Introduction}

Many scientific applications involve liquids and manipulation of liquids on or across surfaces. One obvious technological example is microfluidics, but others include liquid chromatography and any liquid phase sensor involving attachment or immobilization of biological molecules which alters hydrophobicity or hydrophilicity. The volume of liquid can be high or low with much interest in low volumes for on-chip assays. As the size of a liquid scales down the dominant force becomes the liquid-vapor surface tension, $\gamma_{L V} .{ }^{1}$ When the liquid is on a surface the balance between the surface tension and the solid-vapor and solid-liquid interfacial tensions determines both the equilibrium shape of the liquid surface and the driving force in moving the liquid. ${ }^{1,2}$ What determines these interfacial tensions at a microscopic level is solely the chemistry of the surface, but what determines how these forces act upon a liquid is the topography of the surface. ${ }^{3-5}$ An example of the importance of topography is shown by Fig. 1a-c of small $(\sim 2$ $\mathrm{mm}$ diameter) droplets of water on various copper surfaces. On a flat copper surface the contact angle (the angle tangential to the liquid surface measured from the solid through the liquid) is less than $90^{\circ}$, a hydrophobic coating raises this into the range 115$120^{\circ}$, but texturing the surface and then treating it with the same hydrophobic coating increases it to around $160^{\circ}$. The specific surface used in fig. 1c was created using electrodeposition to produce a combination of slight roughness and a "chocolate chip cookie" texturing with lateral sizes in the $20 \mu \mathrm{m}$ range (Fig. 1d).
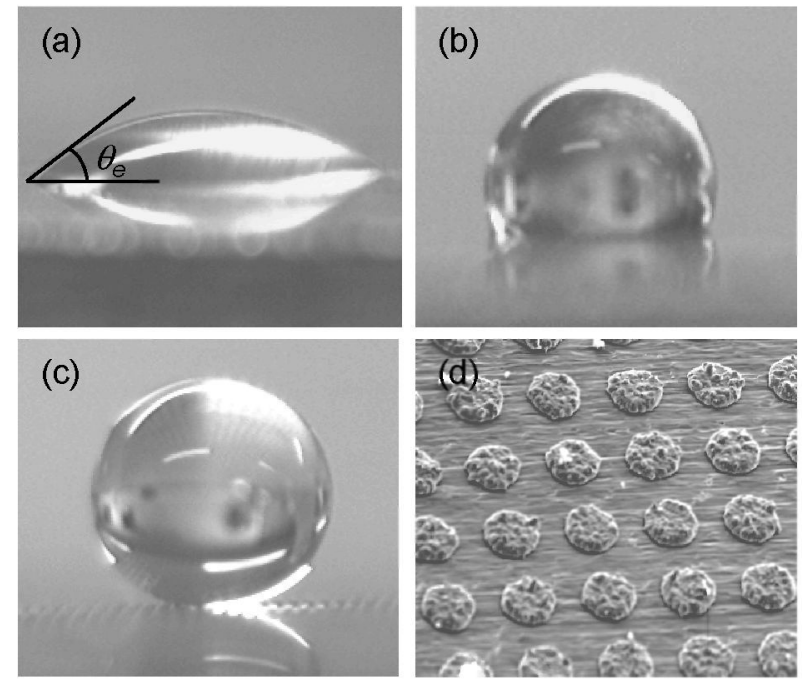

Fig. 1 Water droplets on electrodeposited copper surfaces. (a) flat, (b) flat and hydrophobised, (c) textured and hydrophobised. (d) Electron microscope image of an electrodeposited textured copper surface.
Super-hydrophobicity is defined as water contact angles greater than $150^{\circ}$, but then comes in two distinct flavours with one type providing a "sticky" surface on which liquid drops are difficult to move and the other a "slippy" surface where there is little resistance to drop motion. ${ }^{6}$ Since topography induced effects on contact angles are not limited to water we can refer to super-nonwetting when considering other liquids. On "slippy" superhydrophobic surfaces water drops rolling off the surface also collect dust and so the surfaces are self-cleaning. ${ }^{7-9}$ For some liquids the effect of surface roughness or texture is not to create a rolled-up droplet, but is the opposite effect of super-wetting whereby a droplet is converted into a film. Super-hydrophobic surfaces can be created using roughness or texture at length scales from the sub-micron to the tens of micron and by techniques as diverse as vacuum deposition of PTFE thin films ${ }^{10}$, plasma polymerization $^{11}$, tailoring sol-gel porous materials ${ }^{12,13}$, crystallizing wax ${ }^{14,15}$, electrochemical etching or deposition, and lithography ${ }^{96}$.

\section{Topography Enhanced Wetting}

The principles of super-hydrophobicity were outlined decades ago by Wenzel ${ }^{3}$ and by Cassie and Baxter., ${ }^{4,5}$ Wenzel noted that if a surface was rough and the contacting liquid followed the contours of the surface then the effect of roughness should be to emphasise the intrinsic wetting tendency of either film formation or of roll-up of the liquid. The observed contact angle on such a surface is given by Wenzel's equation,

$$
\cos \theta_{e}^{W}=r \cos \theta_{e}
$$

where the roughness $r>1$ is the ratio of the true surface area of the solid to its horizontal projection and $\theta_{e}$ is the equilibrium contact angle on a smooth flat surface of the same material. The chemistry lies within $\theta_{e}$ whilst controlling the effect of the chemistry on a liquid lies within $r$.

An analogy to Wenzel's equation is an electronic amplifier whereby both a gain (analogue electronics) and the possibility of saturation into one of two states (digital electronics) are possible. Wenzel's equation predicts that the change in observed contact angle on a rough surface $\Delta \theta_{e}^{W}$ due to a change $\Delta \theta_{e}$ induced by surface chemistry is given by,

$$
\Delta \theta_{e}^{W}=r\left[\frac{\sin \theta_{e}}{\sin \theta_{e}^{W}}\right] \Delta \theta_{e}
$$

Thus, the effect of a change in a contact angle due to a surface chemistry change is amplified by the rough surface into a larger change in the observed contact angle. For surface chemistry giving precisely $\theta_{e}=90^{\circ}$ the amplification factor is equal to the 
roughness factor $r$ and this is also approximately true for a wide range of contact angles around $90^{\circ}$. For large enough roughness, Wenzel's equation predicts saturation to either a film $\left(\theta_{e}^{W}=0^{\circ}\right)$ or a complete roll-up of the liquid $\left(\theta_{e}^{W}=180^{\circ}\right)$ should occur. The boundaries between the amplification and the saturation behaviours is given by $\cos \theta_{e}= \pm 1 / r$. From this perspective there is analytical potential for either transducing small changes in liquid properties by amplification of small effects on the surface tension or for producing simple yes/no threshold indicators using the saturation into either a film or a rolled-up droplet.

Due to capillary forces liquids can find it difficult to penetrate into the roughness or texture of a surface. ${ }^{6}$ Energetically it can be favourable for a liquid to break Wenzel's assumption that the surface contours are followed and instead to bridge across the tops of surface protrusions so that the droplet sits upon a composite surface of the solid tops and the air gaps. The observed contact angle is then given by a weighted average of the cosines of the contact angles on the two types of surface (solid and air) using the Cassie-Baxter equation as,

$$
\cos \theta_{e}^{C}=\varphi_{s} \cos \theta_{e}-\left(1-\varphi_{s}\right)
$$

where $\varphi_{s}<1$ is the fraction of the surface of the tops of the solids and $\left(1-\varphi_{s}\right)$ is the fraction that corresponds to the air gaps; the liquid-air contact angle is $180^{\circ}$ and the cosine of this gives the overall minus sign in the second term of the equation. Unlike the Wenzel form of topographic enhancement of hydrophobicity, a complete roll-up of a droplet cannot be achieved with surfaces whose chemistry provides $\theta_{e}<180^{\circ}$. In the Cassie-Baxter form of super-hydrophobicity, a remnant of the surface chemistry determined contact angle, $\theta_{e}$, is always present in the observed contact angle. Cassie-Baxter's equation implies that contact angle enhancement towards super-hydrophobic can be created by surface roughness or texture, but the condition $\varphi_{s}<1$ means amplification of small changes in contact angle, $\Delta \theta_{e}$, does not occur.

Wenzel and Cassie-Baxter provide two alternative views of how a rough or textured surface may influence the wetting behaviour of a surface. The trend observed in experiments on superhydrophobicity is that as texturing or roughness is increased Wenzel enhancement occurs first, followed by a transition to the Cassie-Baxter form of super-hydrophobicity with contact angles of $150^{\circ}-170^{\circ}$. To illustrate this consider the inset in fig. 2 which shows a textured surface consisting of $15 \mu \mathrm{m}$ diameter SU-8 photoresist pillars arranged in a square lattice with pillars spaced every $30 \mu \mathrm{m}$. When the pillars are sufficiently high the effect of the texture is to change some liquids from partial wetting to complete wetting (film formation), consistent with Wenzel's equation, whereas for others the effect is to increase the contact angle in a Cassie-Baxter style (Fig. 2). This Cassie-Baxter form can be promoted as a metastable state by sharp edges on surface protrusions. Such metastable states can be converted to the Wenzel state with lower angles by applying pressure to the liquid. ${ }^{6}$ Knowing which surface has been achieved is a straightforward exercise of tilting the surface and watching whether a droplet of water rolls-off. If the drop rolls at low tilt angles the surface is behaving in the "slippy" Cassie-Baxter form, but if a high tilt angle is required the surface is behaving in the "sticky" Wenzel form. Indeed, if a syringe is used to deposit droplets, a "slippy" surface will be self-evident from the inability to detach a droplet from the needle using light contact with the surface.

\section{Liquid Motion}

A contact angle provides a force per unit length of contact line and so can determine liquid motion. ${ }^{1,2}$ In the capillary rise of a thread of liquid in a tube the height gained, $H$, is determined by the equilibrium contact angle. The capillary driving force is

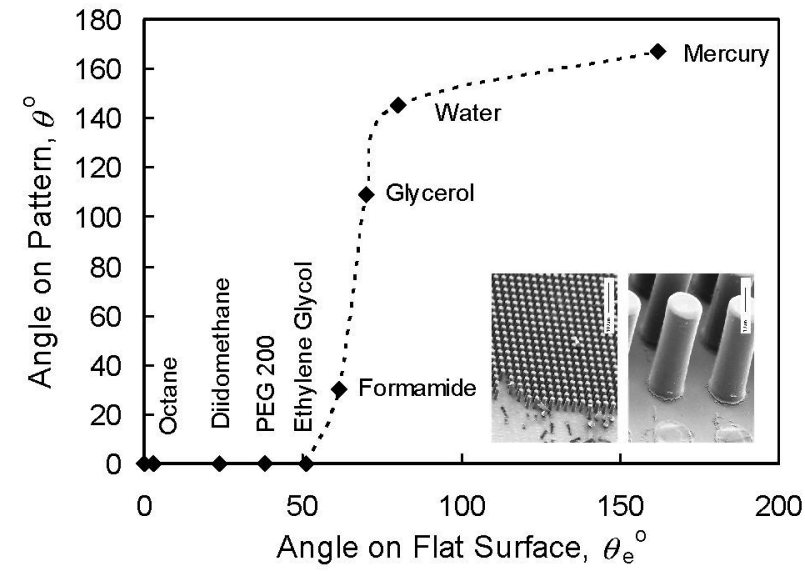

Fig. 2 Contact angles for a range of liquids on a textured SU-8 surface. The inset shows scanning electron microscope images of the lithographically structured surfaces showing a square lattice of $15 \mu \mathrm{m}$ diameter cylindrical pillars with a $30 \mu \mathrm{m}$ lattice parameter (view of a field of pillars and a close-up view of the pillars).

proportional to $\gamma_{\mathrm{L}} \cos \theta_{\mathrm{e}}$ and the length of the three phase solidliquid-vapor contact line, $2 \pi a$, where $a$ is the radius of the tube. The gravitational force on the thread is proportional to the mass of the thread which is proportional to the volume of the thread $\pi a^{2} H$. Assuming a smooth surface, the height gained is $H=\left(2 \kappa^{-2} / a\right) \cos \theta_{\mathrm{e}}$ where $\kappa^{-1}=\left(\gamma_{L_{V}} / \rho g\right)^{1 / 2}$ is the capillary length, $\rho$ is the density of the liquid and $g=9.81 \mathrm{~ms}^{-2}$ is the acceleration due to gravity. A Wenzel form of a surface predicts an amplification, $r$, of the height of capillary rise equal to the roughness factor $r$ of the surface. The Cassie-Baxter form predicts either a reduced rise or even a depression of the thread of liquid.

The contact angle is also fundamental in determining the ease of generating drop motion. When pushing liquid into a drop resting on a surface the maximum contact angle achieved before the drop edge advances, $\theta_{a}$, is larger than the minimum contact angle achieved before the drop edge recedes, $\theta_{r}$, when withdrawing liquid from the drop. Because of contact angle hysteresis, a drop of rain on a window pane will cling without moving until its volume achieves a critical size. For the drop of rain clinging to a window pane there is a higher contact angle, $\theta_{a}$, at the lower side compared to the upper side, $\theta_{r}$. The net surface tension forces on the drop balancing its weight are proportional to $\gamma_{L} r\left(\cos \theta_{r}-\cos \theta_{a}\right)$ and contact angle hysteresis prevents any motion of the drop until it reaches a critical size; the drop then runs down the window pane. ${ }^{6}$ A super-hydrophobic surface of the CassieBaxter type has low hysteresis and so drops can be moved on these surfaces by use of low tilt angles or low driving forces. Interestingly, as a drop rolls it collects dust and debris and so cleans the surface.

Another consideration is the effect of topography on the spreading of out of equilibrium droplets and liquids. During spreading the forces at the contact line are not in balance and the unresolved horizontal force $\gamma_{L V}\left(\cos \theta-\cos \theta_{e}\right)$ drives the spreading. Wenzel type roughness or surface texture causes a modified force $\gamma_{L V}\left(\cos \theta-\cos \theta_{e}\right)$ and the effect is to increase the speed with which a wetting liquid forms a film. ${ }^{17,18}$

\section{Existing and Developing Applications}

Most of the existing research on super-hydrophobic surfaces is materials oriented with a focus on combining super-waterrepellent properties with good optical transparency and wear resistance. ${ }^{19}$ Exterior applications include coatings for radar domes, satellite dishes and glass. These coatings are water repellent, prevent adhesion of snow and provide self-cleaning 
under the action of rain; the mimicking of the self-cleaning action of the Lotus leaf is the so-called Lotus-Effect ${ }^{\mathbb{B}}$. Sol-gel based super-hydrophobic protective coatings are also being used to seal moisture sensitive electronic and optoelectronic components. A super-hydrophobic coated tip has also been suggested to enable accurate dispensing of droplets of low surface tension organic solvents. ${ }^{20}$ The difficulties with superhydrophobic coatings are poor abrasion, chemical and UV resistance, clogging by dust and oil and optical clarity.

There are few research publications on analytical applications of super-wetting and super-hydrophobicity, although there are a number of industrial interests. One theme is based on the idea that any mechanism that can act to change a contact angle at the three phase (solid-liquid-vapor) contact line or which can create a difference in contact angles between two sides of a droplet is a means to actuate motion of the liquid. A driving force can be generated at the edge of the liquid through electrostatic induced changes in the contact angle. ${ }^{21}$ Systems for both droplet actuation $^{22}$ and for motion of a liquid in microcapillaries ${ }^{23}$ have been demonstrated. The basic principle in each case is that a driving force proportional to the change in $\cos \theta_{e}$ can be generated. A comprehensive microfluidics system based on this electrostatic principle and which includes droplet transport, separation and mixing is now commercially available (Nanolytics). A modification of this system using a superhydrophobic surface for easier droplet actuation has been reported. ${ }^{24}$ Problems with this method include the electrostatic pressure causing droplets to convert to the Wenzel "sticky" form of super-hydrophobicity and so preventing motion, and attachment of biological moieties to the surface destroying the tailor made hydrophobic properties. Other applications include an intravenous super-hydrophobic filter to remove microorganisms and particles from emulsions and super-waterrepellent membranes for the separation of pure liquids from mixtures based on surface tension differences. ${ }^{25,26}$ Currently Bell laboratories are working on a tunable super-hydrophobic silicon nanograss with potential applications in micro-fluidics, chemical microreactors and bio-chemical detection, but few details are available. In the super-hydrophilic case, the main application under consideration appears to be anti-fogging for items such as mirrors.

\section{Potential Analytical Applications}

The low-tilt and low force required to move a droplet on a "slippy" surface of the Cassie-Baxter type has been the basis for a patent on a toy and the idea has been extended to toys with tracks based on super-hydrophobic surfaces. ${ }^{27}$ In a similar manner, creating tracks on surfaces for microfluidics applications by varying the roughness of texture across a surface may be feasible. If a widely spaced super-hydrophobic surface texture is surrounded by a more narrowly spaced superhydrophobic surface texture a drop will tend to roll into the area with the more widely spaced texture. The variation in texture will lead to a patterning of the effective surface free energy and hence can be used to define regions and paths on the surface.

In biological applications, one difficulty with specifically tailored surfaces is that attachment or immobilization of biological moieties on the surface immediately changes the chemical or topographic properties of the surface and may remove the super-hydrophobic properties. However, it is conceivable that this problematic feature could be used constructively in a new type of sensor for studying interactions such as antigen-antibody, nucleic acid hybridization and molecular receptor-ligand reactions. ${ }^{28}$ For example, a shear wave acoustic wave sensor (quartz crystal microbalance or surface acoustic wave device) has a resonant frequency which changes when mass deposition or attachment to the surface from the liquid occurs. ${ }^{29,30}$ The surface of the sensor could be made superhydrophobic in the "slippy" form so that incomplete liquid penetration occurs. Any deposition or binding event which reduced the hydrophobicity and so removed the superhydrophobicity, would cause the liquid to enter the surface texture. This would generate a transducer response much larger than that due to the mass of the binding molecule. Even without binding or mass deposition, a hydrophobic coating could be selected to allow changes in the surface tension of the liquid to result in a loss of the hydrophobic (non-wetting) properties of the surface towards the liquid. Another possible area for consideration is the use of super-wetting to distinguish between liquids which are partially wetting. If the liquids are placed on a series of surfaces with increasing texture or roughness a critical length scale could be identified which converts them into complete wetting liquids. A variation on this theme could involve measuring the enhancement of capillary rise caused by roughness or surface texture.

\section{Conclusion}

Understanding how surface topography or texture combines with surface chemistry to determine the wetting of surfaces by liquids is important in any application involving small volumes of liquids where capillary effects are dominant. The most spectacular example of this combination is the creation of superhydrophobic surfaces from hydrophobic surfaces, but the principles are not restricted to water and are equally applicable to problems involving film formation and movement of liquids on surfaces. The major focus of research and applications development has been in the most obvious uses of superhydrophobicity as water-repellent, self-cleaning and resistance/friction reducing coatings. However, it is probable that a wider range of analytical applications will exist based on the super-non-wetting and the super-wetting properties of topographically structured surfaces.

\section{Acknowledgement}

The authors' acknowledge the financial support of the UK EPSRC and MOD/Dstl (Grants GR/R02184/01 and GR/ GR/S34168/01). They would also like to thank G. Chabrol, S. Aqil, C. Evans and C.C. Perry.

\section{References}

1 P.G. de Gennes, Rev. Mod. Phys., 1985, 57, 827

2. L. Léger and J.F. Joanny, Rep. Prog. Phys., 1992, 55, 431

3. R.N. Wenzel, Ind. Eng. Chem., 1936, 28, 988; J. Phys. Colloid Chem., 1949, $\mathbf{5 3}, 1466$.

4. A.B.D Cassie and S. Baxter, Trans. Faraday Soc., 1944, 40, 546.

5. R. E. Johnson and R. H. Dettre, in Contact angle, Wettability and Adhesion, Advances in Chemistry Series, 1964, 43, 112.

6. D. Quéré, A. Lafuma and J. Bico, Nanotechnology, 2003, 14, 1109.

7. C. Neinhuis and W. Barthlott, Annals of Botany, 1997, 79, 667.

8. D. Richard and D. Quéré, Europhys. Lett., 1999, 48, 286.

9. P. Aussillous and D. Quéré, Nature, 2001, 411, 924.

10. J.D. Miller, S. Veeramasuneni, J. Drelich, M.R. Yalamanchili, and G. Yamauchi, Poly. Eng. Sci., 1996, 36, 1849.

11. S.R. Coulson, I. Woodward, J.P.S. Badyal, S.A. Brewer and C. Willis, J. Phys. Chem., 2000, B104, 8836.

12. K. Tadanaga, N. Katata, N., and T. Minami, J. Am. Ceram. Soc., $1997,80,1040$.

13. N. J. Shirtcliffe, G. McHale, C.C. Perry and M.I. Newton, Langmuir, 2003, 19, 5626 .

14. T. Onda, S. Shibuichi, N. Satoh and K. Tsujii, Langmuir, 1996, 12, 2125 .

15. S. Shibuichi, T. Onda, N. Satoh and K. Tsujii, J. Phys. Chem., 1996, 100, 19512

16. D. Öner, T. McCarthy, Langmuir, 2000, 16, 7777.

17. G. McHale and M. I. Newton, Colloids and Surfaces, 2002, A206, 193. 
18. G. McHale, N.J. Shirtcliffe, S. Aqil, C.C. Perry and M.I. Newton, Phys. Rev. Letts., 2003, 93, art. 036102.

19. R. Blossey, Nature Materials, $2003,2,301$.

20. P.A. Materna, Method and apparatus for dispensing small volume of liquid, such as with a wetting-resistant nozzle, US Patent Application 20020084290, 2002.

21. M. Vallet, B. Berge and L. Vovelle, Polymer, 1996, 37, 2465.

22. M.G. Pollack, R.B. Fair and A.D. Shenderov, Appl. Phys. Lett., 2000, 77,1725 .

23. M. W. J. Prins, W. J. J. Welters and J. W. Weekamp, Science, 2001, 291, 277.

24. A. Torkkeli, A. Haara, J. Saarilahti, H. Harma, T. Soukka and P. Tolonen, Droplet manipulation on a super-hydrophobic surface for microchemical analysis, Proceedings of Transducers 01/Eurosensors XV 475 and $1150,2001$.

25. S.-B. Lee, I-J Park, K.-W. Lee, D.-K. Kim, J.-H. Kim, J.-W. Ha, H.S. Park, D.-H. Jung and J. Ruehe, Super water-repellent organic/inorganic composite membrane, US Patent Application $20030080049,2003$.

26. S. N. Buhl, S. I. Ahmad and A. Grassick, Apparatus and method for the separation of immiscible liquids, U.S. Patent 4,971,912, 1990.

27. F.G. Reick, Toys and games using super-hydrophobic surfaces, U.S Pat. No. 4,199,142, 1980; Water maze game with super-hydrophobic surface, U.S. Patent No. 4,142,724

28. G. McHale, M.I. Newton and M. Thompson, Acoustic biosensor, Canadian "informal" patent application CA2451413, 2003. (filed 28/11/2003; published 28/5/2005)

29. Ballantine, D.S., White, R.M., Martin, S.J., Ricco, A.J., Zellers, E.T., Frye, G.C., and Wohjlten, H., "Acoustic wave sensors" (Academic, New York, 1997)

30. Thompson, M., and Stone, D.C., "Surface-launched acoustic wave sensors" (John Wiley and Sons, New York, (1997). 